\title{
A Novel Wideband Bandpass Filter using H-shaped DGS
}

\author{
Van-Phuong Do, Duy-Manh Luong, Chi-Hieu Ta, and Minh-Tan Doan
}

Department of Radio Electronic Engineering, Le Quy Don Technical University, Hanoi, Vietnam

\begin{tabular}{l} 
Article Info \\
\hline Article history: \\
Received October 4, 2017 \\
Revised March 14, 2018 \\
Accepted: April 2, 2018 \\
\hline
\end{tabular}

Keyword:

Bandpass filter

defected ground structure

DGS unit

H-shaped DGS

Wideband bandpass filter

\begin{abstract}
This paper presents a novel compact wide-band bandpass filter (BPF) having good selectivity. It is designed using a dual-plane structure which consists of a parallel-coupled microstrip line on the upper surface and three $\mathrm{H}$-shape defected ground structure (DGS) on the ground plane. By adding three H-shape DGS units on the ground plane, then properly adjusting their dimensions and position, the bandwidth and selectivity of the designed filter can be significantly improved. A compact prototype of wide-band microstrip bandpass filter has been designed, fabricated and measured to apply for the wireless systems. The filter shows a center frequency at $4.8 \mathrm{GHz}$, passband from $2.8 \mathrm{GHz}$ to $6.8 \mathrm{GHz}$ with maximum insertion loss and return loss of $0.8 \mathrm{~dB}$ and $40 \mathrm{~dB}$, respectively. The measured results agrees well with the theoretical expectations validating the proposed design.
\end{abstract}

Copyright (c) 2018 Institute of Advanced Engineering and Science. All rights reserved.

\section{Corresponding Author:}

Name: Van-Phuong Do

Affiliation

Address: 236, Hoang Quoc Viet, Hanoi, Vietnam

Phone: 0995155168

Email: dvphuongntcity@gmail.com,phuongdovan@tcu.edu.vn

\section{INTRODUCTION}

Recently, the microstrip wide-band bandpass filter (BPF) with high performance has become one of the most important circuit components in the modern broadband wireless communication systems [1]. Furthermore, it has received much attention due to the promising advantages such as high selectivity, small size, low cost and easy fabrication. In [2], by adjusting dimensions of the meandered transversal resonator and asymmetrical interdigital coupled lines, a wideband microstrip BPF with good rejection in out of the band has been designed and fabricated. However, these filters have a major disadvantage of large size. In [3], another wide-band bandpass filter using the folded multiple-mode resonator is designed. However, this filter has a drawback of weak isolation between the input and output. In [4], [5] authors an application of the DGS to can significantly improve the reflection coefficient $\left(S_{11}\right)$ of microstrip antennas. For microstrip bandpass filter design, In [6], [7], the complementary splitring resonator (CSRR) is etched on the ground plane. It is used as the basic resonant unit to design the wideband bandpass filter with good wideband response. However, the filter of this type is either quite large in size or has a complex structure. In [8], the method to design the UWB bandpass filter using a transmission line structure with two embedded U-shape slots and a dumbbell shape DGS periodic array etched on the ground in order to obtain the wide stopband effect but this filter is still bulky. It has a relatively larger size, up to $24 \mathrm{~mm}$ in length. In [9], a novel wideband bandpass filters with compact size and low insertion loss using DGS slots were designed and analyzed. In [10], an UWB BPF with high selectivity was proposed. Its design consists of a high-low impedance microstrip line, a short-circuited stub combined with different DGSs (H-shape DGS slots and dumbbell-shape DGS slots). However, this type of filters is also large in size with a relatively complicated structure.

In this paper, we present a technical solution to the abovementioned drawbacks of the bandpass filter by using the H-shaped DGS units for wide-band filter design with the aim of improving its performance in passband and stopband characteristics. A novel compact wide-band microstrip bandpass filter using H-shaped DGS using a parallel-coupled microstrip line resonator on the upper surface combined with three H-shaped DGS units on the ground plane is designed and fabricated. The theoretical design, simulation and experimental results of the filter are presented and discussed. 


\section{RESONANT PROPERTY OF H-SHAPED DGS}

The DGSs is etched on the ground plane of the planar filter, they disturb the shield current distribution on the ground plane leading to the change in characteristics of the resonant circuit in the upper plane. This structure can suppress the harmonics, reduce the physical dimensions and improve stopband and passband characteristics of the microstrip filter [11]. There are many available DGSs with different shapes as presented in [12], [13]. Where, the dumbbell-shaped DGS is structured very simply and can be easily adjusted its geometry dimensions to tune the characteristics of the resonance circuits. The structure of a dumbbell-shaped DGS unit comprises two rectangular etched defects with the dimensions of $(a \times b)$ in backside metallic ground plane. They are connected to each other by a narrow slot with the dimensions of $(g \times d)$ as shown in Figure 1(a).

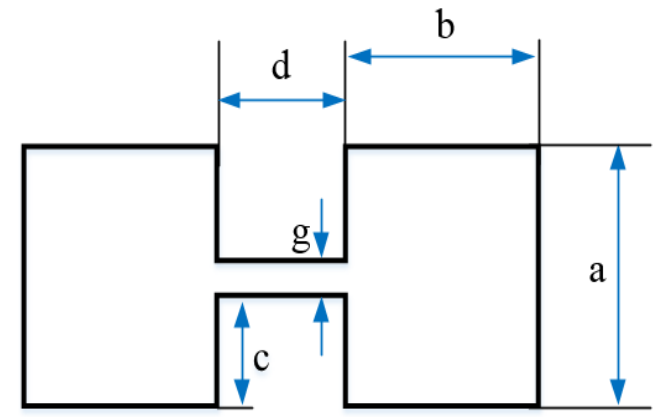

(a)

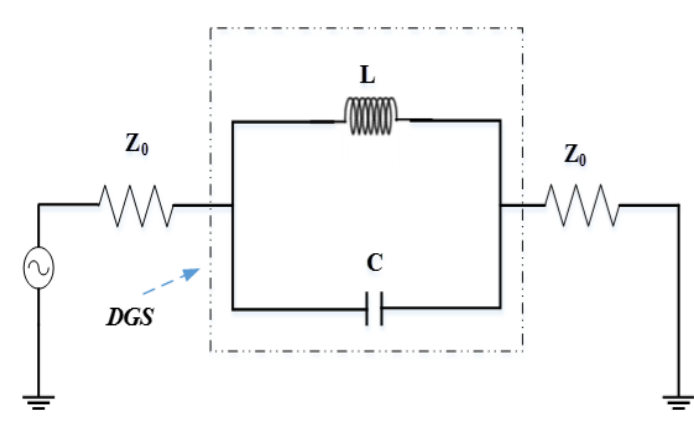

(b)

Figure 1. The structure and the equivalent circuit of DGS dumbbell-shaped. (a) Structure. (b) Equivalent circuit [11].

By changing the geometry of the rectangular etched defects, one can change the inductance of the dumbbell shaped DGS unit while changing the narrow slot size, its capacitance can be varied [14]. Therefore, the dumbbell shaped DGS unit can be treated as a parallel $L C$ resonant circuit as indicated in Figure 1(b). Its equivalent $L$ and $C$ values are determined as below.

$$
\begin{gathered}
L=\frac{1}{4 \pi^{2} f_{0}^{2} C} \\
C=\frac{f_{C}}{4 \pi Z_{0}\left(f_{0}^{2}-f_{C}^{2}\right)}
\end{gathered}
$$

where, $f_{0}$ is the resonant frequency of the parallel $L C$ resonant circuit, $f_{C}$ is the cutoff frequency of the prototype low-pass filter and $Z_{0}$ denotes the scaled impedance of the in/out terminated ports, and is given by the prototype value of the Butterworth type low pass filter.

It is noted that, since there is no explicit correlation between the physical dimensions and the parameters of the LC equivalent circuit, the performance of the dumbbell-shaped DGS is only predictable through an optimization process. Applying promising properties of the dumbbell-shaped DGS to wide-band BPF design, we propose a novel compact wide-band BPF using H-shaped DGSs. The detailed design process will be presented in the following sections.

\section{ANALYSIS AND DESIGN OF PROPOSED FILTER}

To design the proposed wide-band microstrip bandpass filter we first design a parallel-coupled microstrip line resonator on the upper plane as indicated in Figure 2(a). It is composed of two microstrip transmission-line sections worked as half-wavelength resonators. In this case, the two resonant frequencies may be overlapped or split depending on the geometrical conditions of the structure. The stronger coupling can be achieved when narrowing the distance $S$. The energy then will be coupled to the output forming a passband, as depicted in Figure 2(b). The dimensions of the parallel-coupled microstrip line resonator are set as $L_{1}=2.1 \mathrm{~mm}, L_{2}=9.7 \mathrm{~mm}, W_{1}$ $=1.4 \mathrm{~mm}, W_{2}=0.6 \mathrm{~mm}$ and $S=0.38 \mathrm{~mm}$. However, the distance $S$ cannot be too narrow and the couple line cannot form transmission if $S$ is too larger. This means there should be an optimum value for $\mathrm{S}$. 


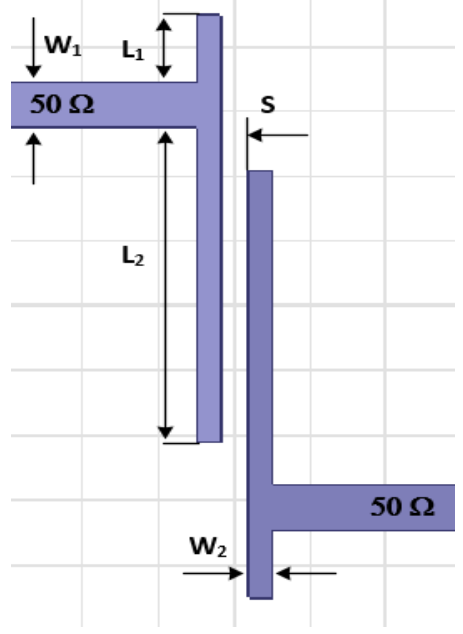

(a)

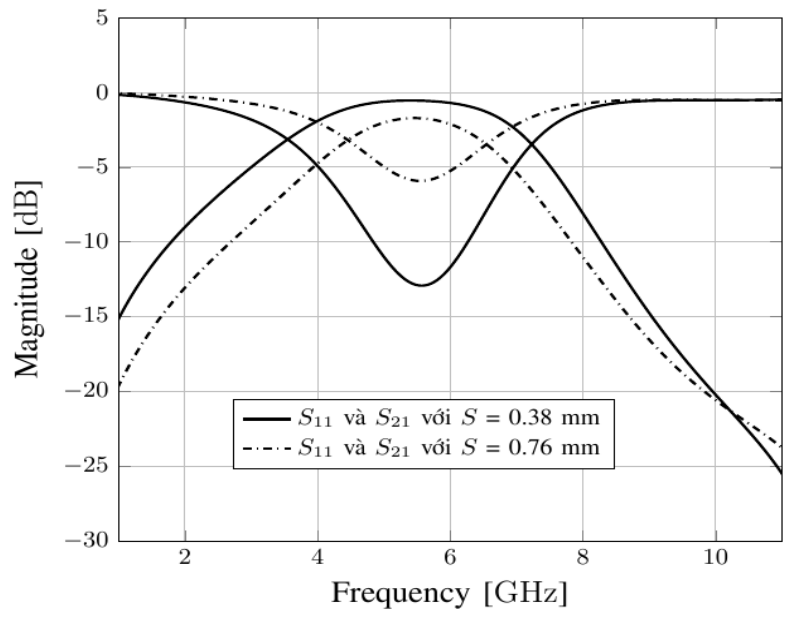

(b)

Figure 2. Layout and response of the wide-band BPF without H-shaped DGS. (a) Layout. (b) Frequency responses.

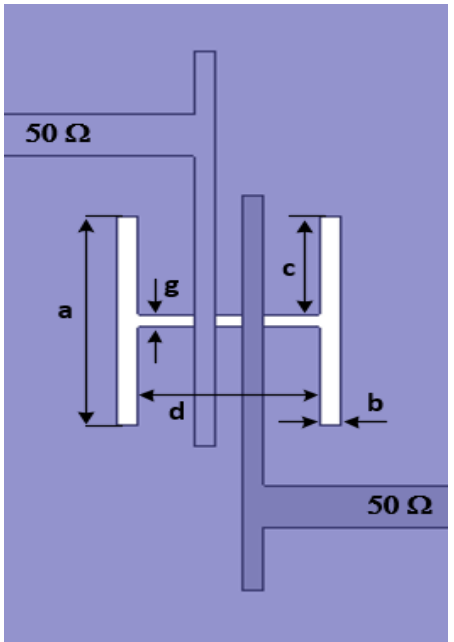

(a)

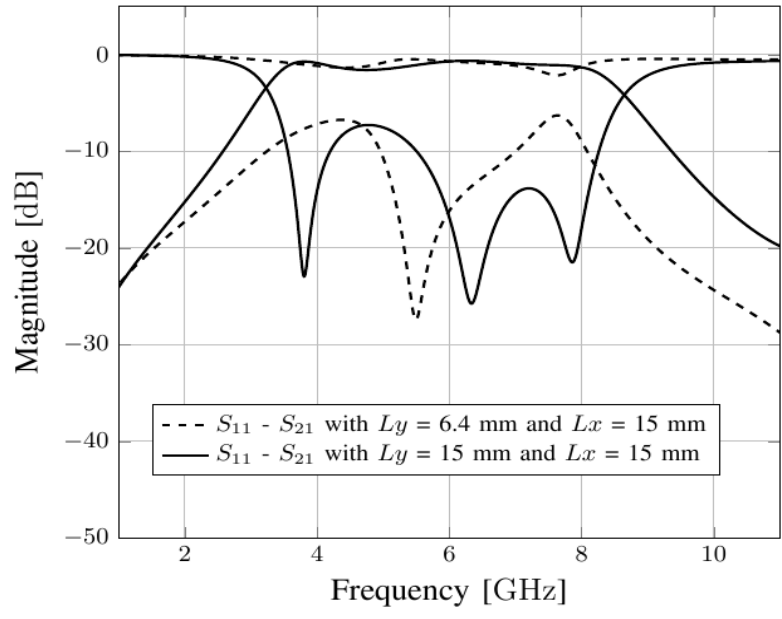

(b)

Figure 3. . Layout and responses of the wide-band micostrip BPF with a H-shaped DGS unit. (a) Layout, (b) Frequency responses. 
To improve the characteristics of parallel-coupled microstrip line resonator on the upper surface, we design an additional H-shaped DGS unit at the center of the ground plane as shown in Figure 3(a). The initial H-shaped DGS model and $L, C$ values are determined by equations (1) and (2).

The dimensions of the H-shaped DGS are designed as $a=7 \mathrm{~mm}, b=0.6 \mathrm{~mm}, d=5 \mathrm{~mm}, g=0.4 \mathrm{~mm}$ and $c=3.3 \mathrm{~mm}$. Since the energy is coupled by the H-shaped DGS, its resonant frequency is close to the center frequency of the passband of the parallel-coupled microstrip line resonator.

The simulated results are shown in Figure 3(b) which is obtained with different H-shaped DGS locations. A position with value of $L_{x}=15 \mathrm{~mm}, L_{y}=15 \mathrm{~mm}$ seems to be good for $S_{11} \mathrm{v} S_{21}$. Nevertheless it can be seen, from these results that the quality of such a proposed filter is limited and not sufficient for wireless telecommunication application.

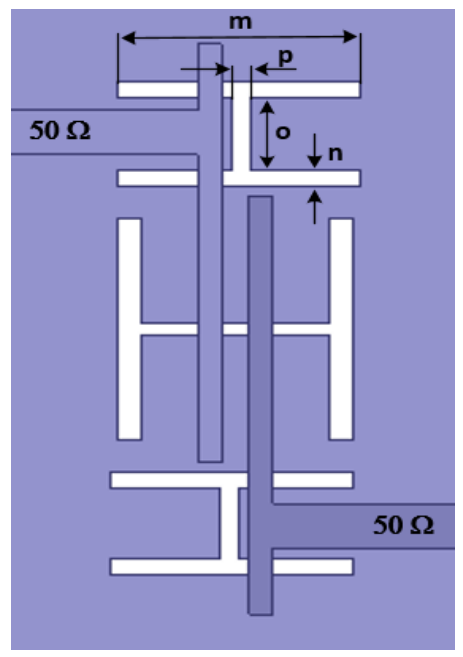

Figure 4. Layout of the proposed wide-band microstrip BPF with three H-shaped DGS units.

To enhance the quality of the proposed filter which meets requirements of the wireless systems, we add two additional $\mathrm{H}$-shaped DGS units on the ground plane as illustrated in Figure 4. This addition will increase the degree of freedom of the tuned parameters for the proposed filter leading to high possibility of perofrmance improvement.

As mentioned before, the resonant frequency and bandwidth of the proposed filter can be adjusted easily by changing the geometric dimensions of three H-shaped DGS units on the ground plane.

We properly adjust the dimensions of three H-shaped DGS units, then the target resonant frequency and bandwidth can be obtained as depicted in Figure 5 and Figure 6, respectively.

Figure 5 shows that when changing dimensions $a$ and $g$ of the central H-shaped DGS unit, desired propagation characteristics of the proposed filter can be achieved. $a$ has an significant effect on the return loss. As the dimensions of $a$ increases from $6 \mathrm{~mm}$ to $8 \mathrm{~mm}$, the return loss varies remarkably. A value of $a=7 \mathrm{~mm}$ should be good. Similarly, a value of $g=0.4 \mathrm{~mm}$ seems to be the good for performance improvement. In this case, the bandwidth can be slightly varied. It is noted that changing in dimensions $b$ and $d$ has no remarkable impact on $S_{11}$ and $S_{21}$ improvement [11]. Besides, the bandwidth also varies slightly.

In addition, Figure 6 indicates that, when adjusting the dimensions $p$ and $m$ of the two side H-shaped DGS units the desired $S_{11}$ can be achieved. Varying $p$ and $m$ has an effect on the return loss and bandwidth. And a pair value of $p=0.5 \mathrm{~mm}$ and $m=7.5 \mathrm{~mm}$ seems to be the good for performance improvement. Changing in $n$ and $o$ of H-shaped DGS has no effect on $S_{11}$ and $S_{21}$ improvement. However, in this case the bandwidth bandpass of the proposed filter gets degraded from $4.15 \mathrm{GHz}$ to $3.58 \mathrm{GHz}$ as shown in Figure 7.

From the above deep investigations, a set of optimized dimensions for the filter can be realized as: the parallel-coupled microstrip line resonator on the upper surface with $L_{1}=2.1 \mathrm{~mm}, L_{2}=9.7 \mathrm{~mm}, W_{1}=1.4 \mathrm{~mm}$, $W_{2}=0.6 \mathrm{~mm}, S=0.38 \mathrm{~mm}$; the H-shaped DGS units at center on the ground plane with $a=7 \mathrm{~mm}, b=0.6 \mathrm{~mm}, d$ $=5 \mathrm{~mm}, g=0.4 \mathrm{~mm}$ and $c=3.3 \mathrm{~mm}$; and two horizontal H-shaped DGS units on the ground plane with $m=7.5$ $\mathrm{mm}, n=0.5 \mathrm{~mm}, o=2.3 \mathrm{~mm}$ and $p=0.5 \mathrm{~mm}$.

Such a filter exhibits a passband center frequency of $4.93 \mathrm{GHz}$, passband bandwidth of $72.6 \%$ (from $3.14 \mathrm{GHz}$ to $6.72 \mathrm{GHz}$ ); within the passband, the insertion loss is less than $0.68 \mathrm{~dB}$ while the return loss is better than $31.5 \mathrm{~dB}$. This superior performance of the designed wide-band bandpass filter with three H-shape DGS units 


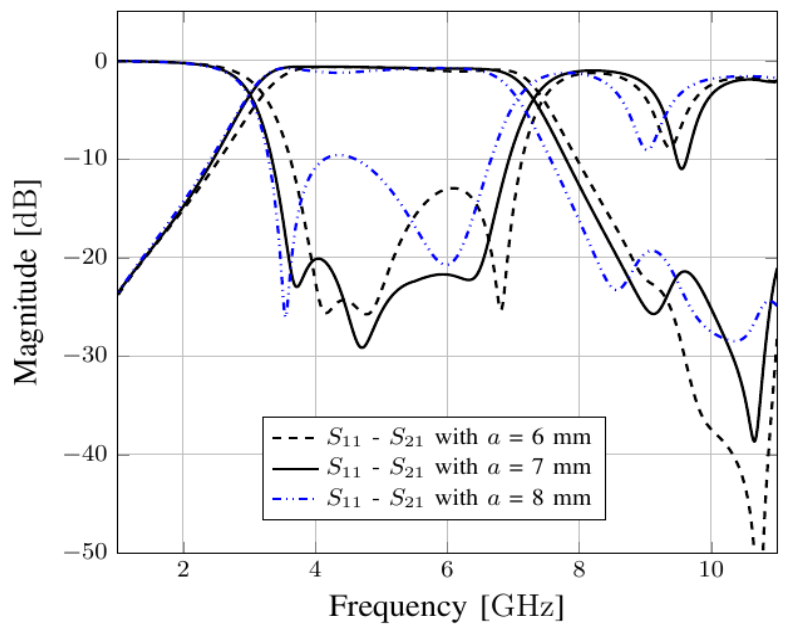

(a)

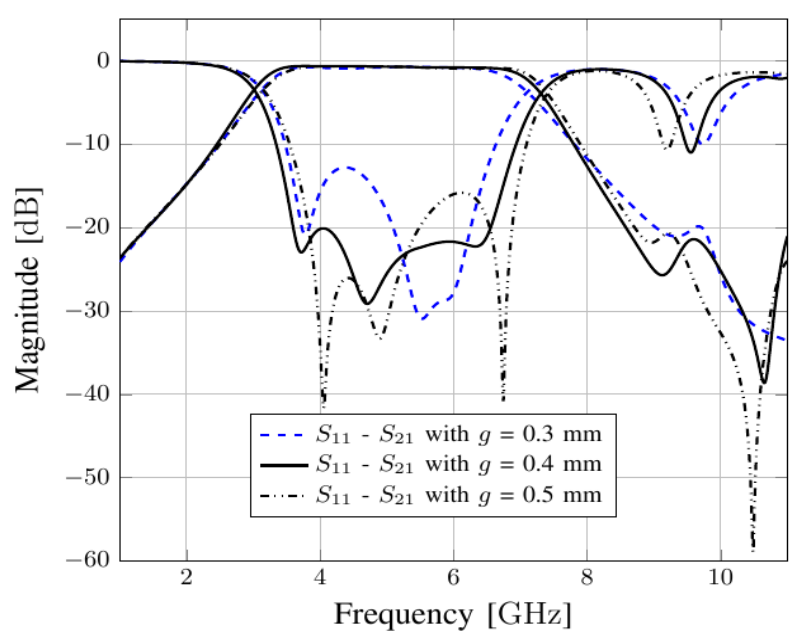

(b)

Figure 5. Simulated frequency responses of the proposed wide-band microstrip BPF with different $a$ and $g$. The dimensions of the center H-shaped DGS unit are $a=7 \mathrm{~mm}, b=0.6 \mathrm{~mm}, d=5 \mathrm{~mm}$ and $g=0.4 \mathrm{~mm}$.

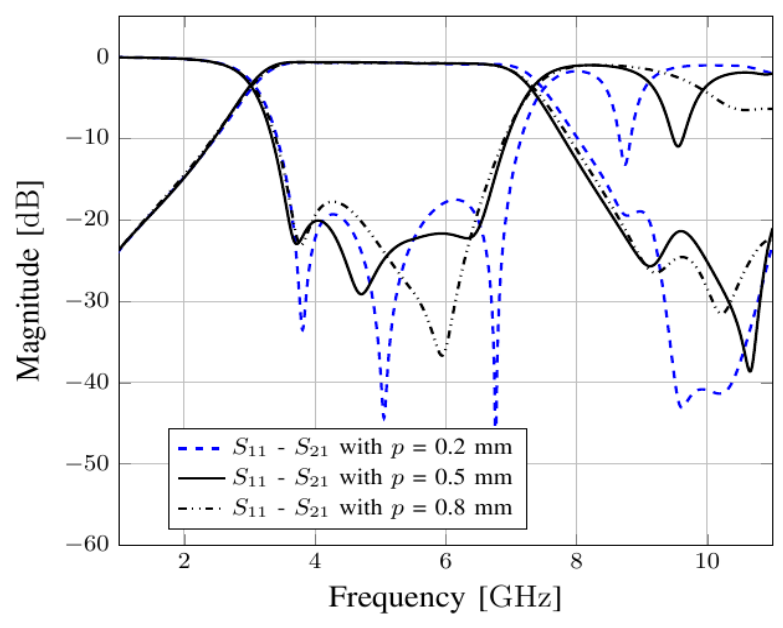

(a)

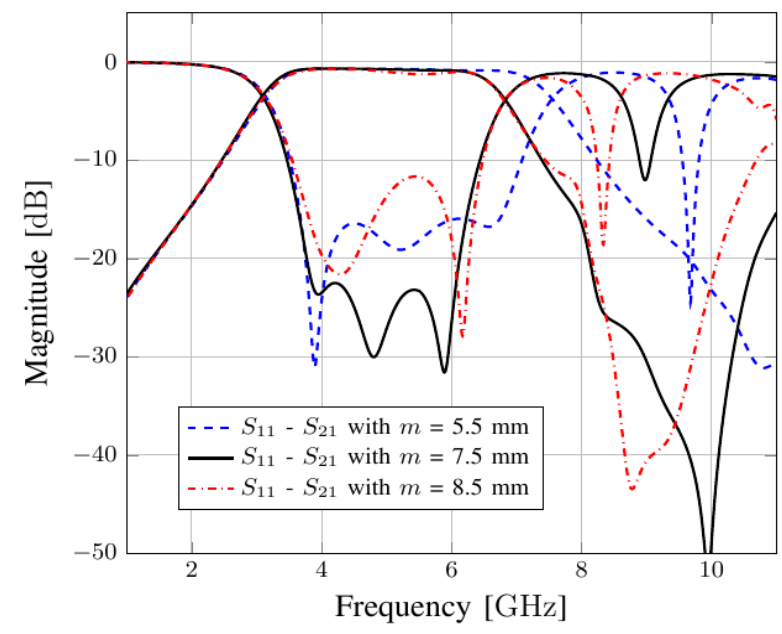

(b)

Figure 6. Simulated frequency responses of the proposed wide-band microstrip BPF with different $p$ and $m$. The dimensions of the upper and lower H-shaped DGS unit are $m=7.5 \mathrm{~mm}, n=0.5 \mathrm{~mm}, o=2.3 \mathrm{~mm}$ and $p=0.5 \mathrm{~mm}$. 


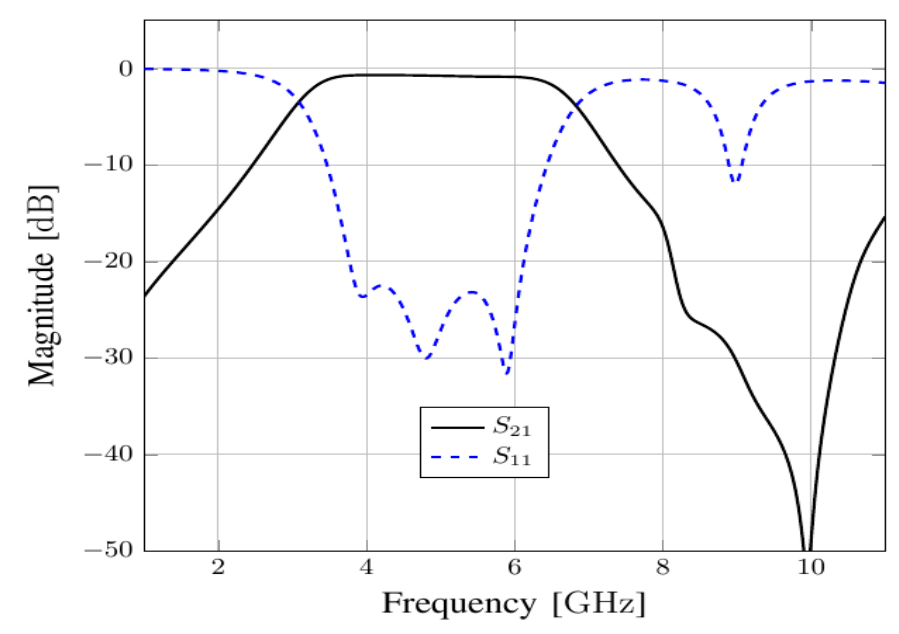

Figure 7. Simulated frequency responses of the proposed wide-band microstrip BPF.

compared to the previously filter without DGS demonstrates validity of the proposed design (especially in terms of the insertion loss, the passband ripple and the $3 \mathrm{~dB}$ bandwidth).

\section{FABRICATION OF THE PROPOSED FILTER AND DISCUSS}

One prototype of the proposed wide-band microstrip bandpass filter using H-shaped DGS has been designed and fabricated on a substrate RO 4350 with $\varepsilon_{r}=3.66$ and $h=0.762 \mathrm{~mm}$.

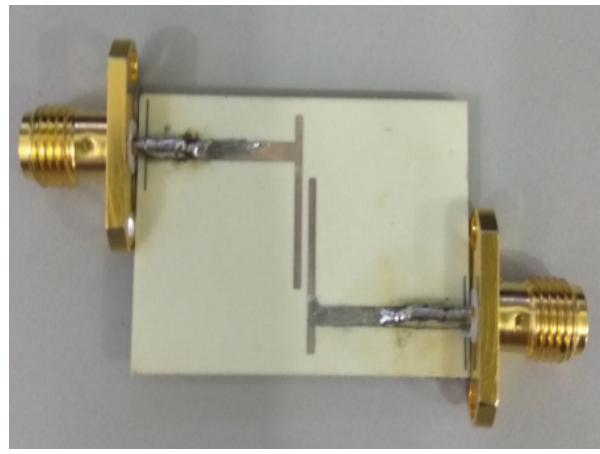

(a)

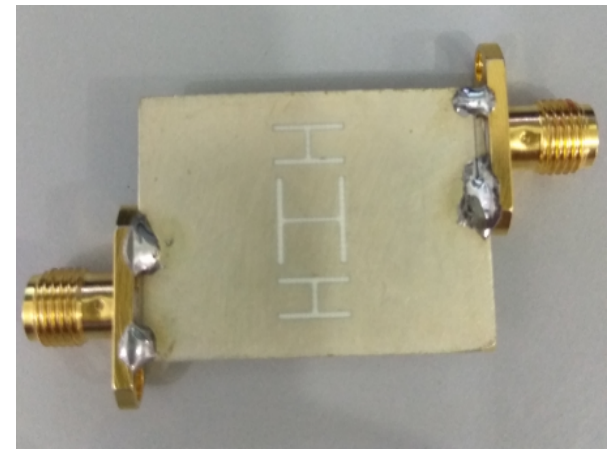

(b)

Figure 8. Photograph of the proposed wide-band microtrip BPF. (a) Top view. (b) Bottom view.

The fabricated filter photograph is shown in Figure 8, the size of the filter is $(18 \times 7.8) \mathrm{mm}$ which is quite compact. The measured results of the fabricated filter are shown in Figure 9, where good agreement between the simulation and measurement can be clearly observed. The measured $3 \mathrm{~dB}$ fractional bandwidth of the fabricated filter achieves $83.3 \%(2.8-6.8) \mathrm{GHz}$. The center frequency is $4.8 \mathrm{GHz}$. The maximum passband insertion loss is $0.8 \mathrm{~dB}$ and the maximum return loss is $40 \mathrm{~dB}$. This measured performance is very promising for the wireless communications application. The small deviations between the simulated and measured results is considered due to our poor soldering craft and error in fabrication or measurement system.

\section{CONCLUSION}

This paper presents a novel technical solution for improvement of wide-band bandpass filter performance in the stopband and passband by using the H-shaped DGS units. A compact wide-band microstrip BPF using parallel-coupled microstrip line resonator and three H-shape DGS units has been designed and fabricated. The measured results show that the filter works well at the frequency range of $2.8 \mathrm{GHz}$ to $6.8 \mathrm{GHz}$. The maximum 


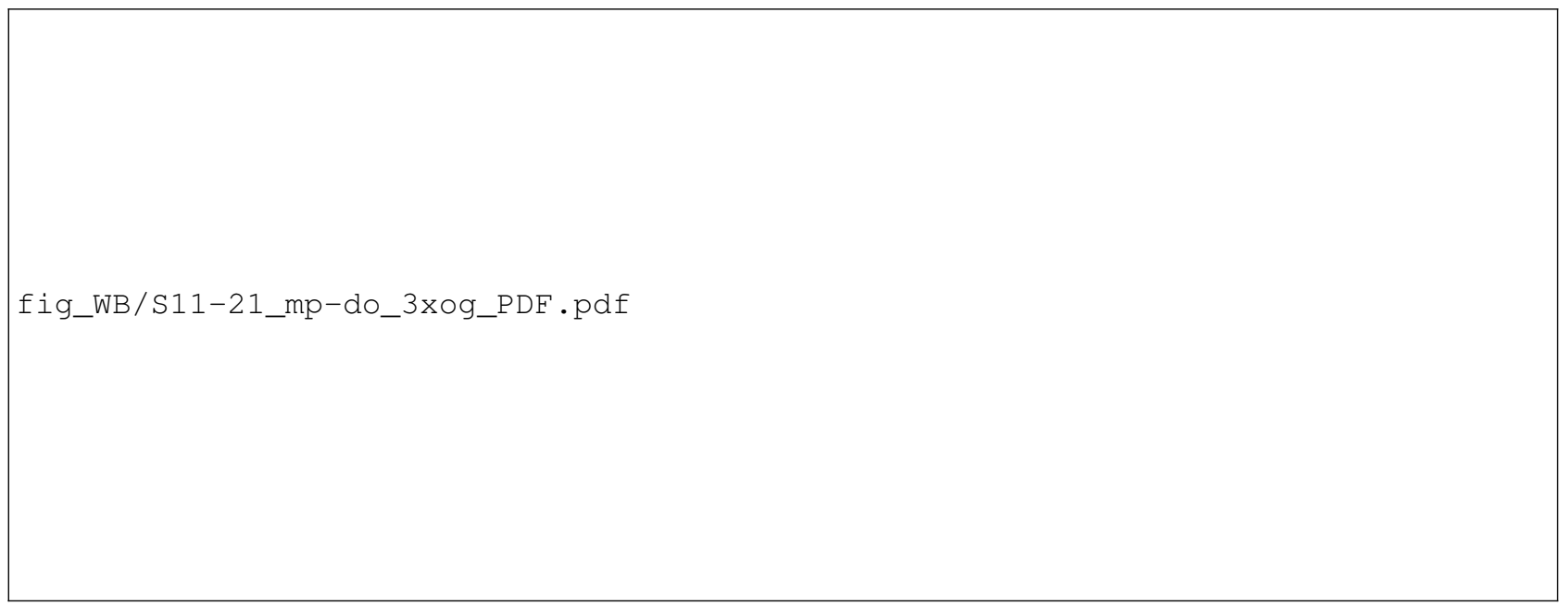

Figure 9. Simulated and measured responses of the proposed wide-band microstrip BPF.

passband insertion loss is $0.8 \mathrm{~dB}$ and the maximum return loss is $40 \mathrm{~dB}$. Therefore, the filter fits well for various wireless communication systems such as microwave Access (WiMAX) at $3.5 \mathrm{GHz}$, INSAT C-band at $4.6 \mathrm{GHz}$, Wireless Local Area Network (WLAN) at $5.6 \mathrm{GHz}$ applications. Very good agreement between simulations and measurements demonstrates validity of the prosed design strategies.

\section{REFERENCES}

[1] M. Nosrati and M. Mirzaee, "Compact wideband microstrip bandpass filter using quasi-spiral loaded multiplemode resonator," IEEE Microw. Wireless Compon. Lett., vol. 20, pp. 607-609, 2010.

[2] S. Sun, L. Zhu, and H.-H. Tan, "A compact wideband bandpass filter using transversal resonator and asymmetrical interdigital coupled lines," IEEE Microw. Wireless Compon. Lett., vol. 18, pp. 173-175, 2008.

[3] H. Wang, Q.-X. Chu, and J.-Q. Gong, "A compact wideband microstrip filter using folded multiple-mode resonator," IEEE Microwave and Wireless Components Letters, vol. 19, pp. 287-289, 2009.

[4] I. Zahraoui, A. Errkik, M. Abounaima, A. Tajmouati, L. Abdellaoui, and M. Latrach, "A New Planar Multiband Antenna for GPS, ISM and WiMAX Applications," International Journal of Electrical and Computer Engineering (IJECE), vol. 7, pp. 2018-2026, 2017.

[5] S. Elajoumi, A. Tajmouati, A. Errkik, A. Sanchez, and M. Latrach, "Microstrip Rectangular Monopole Antennas with Defected Ground for UWB Applications," International Journal of Electrical and Computer Engineering (IJECE), vol. 7, pp. 2027-2035, 2017.

[6] X. Lai, Q. Li, P.-Y. Qin, B. Wu, and C.-H. Liang, ”A novel wideband bandpass filter based on complementary split-ring resonator," Progress In Electromagnetics Research C, vol. 1, pp. 177-184, 2008.

[7] X. Luo, H. Qian, J.-G. Ma, and E.-P. Li, "Wideband bandpass filter with excellent selectivity using new CSRRbased resonator," Electronics letters, vol. 46, pp. 1390-1391, 2010.

[8] G. Yang, R. Jin, and J. Geng, "Planar microstrip UWB bandpass filter using U-shaped slot coupling structure," Electronics Letters, vol. 42, pp. 1461-1463, 2006.

[9] Q. Song, H. Cheng, X. Wang, L. Xu, X. Chen, and X. Shi, "Novel wideband bandpass filter integrating HMSIW with DGS,' Journal of Electromagnetic Waves and Applications, vol. 23, pp. 2031-2040, 2009.

[10] G.-M. Yang, R. Jin, C. Vittoria, V. Harris, and N. Sun, "Small ultra-wideband (UWB) bandpass filter with notched band," IEEE Microwave and Wireless Components Letters, vol. 18, pp. 176-178, 2008.

[11] D. Ahn, J.-S. Park, C.-S. Kim, J. Kim, Y. Qian, and T. Itoh, "A design of the low-pass filter using the novel microstrip defected ground structure," IEEE Tran. Microw. Theo. Techn., vol. 49, pp. 86-93, 2001.

[12] C. Garg and M. Kaur, "A review of defected ground structure (DGS) in microwave design," International Journal of Innovative Research in Electrical, Electronics, Instrumentation and Control Engineering, vol. 2, 2014.

[13] A. Kumar and M. Kartikeyan, "Design and realization of microstrip filters with new defected ground structure (DGS)," Engineering Science and Technology, an International Journal, vol. 20, pp. 679-686, 2017.

[14] A. Kumar and M. Kartikeyan, "Design and realization of microstrip filters with new defected ground structure (DGS)," Engineering Science and Technology, an International Journal, vol. 20, pp. 679-686, 2017. 


\section{BIOGRAPHIES OF AUTHORS}

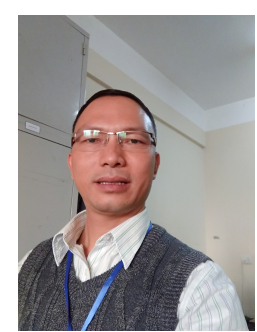

Van-Phuong Do graduated College specialized Telecommunications engineering from Telecommunication University, Nhatrang, Khanhhoa, Vietnam, in 1996. He received the B.Eng and M.Eng degrees in Radio Elictronic Engineering from Le Qui Don Technical University, Hanoi, Vietnam in 2000 and 2003, respectively. He is currently a teacher and pursuing the Ph.D. degree in electronic engineering at Le Quy Don Technical University, Hanoi. His research interest includes the technique of electronic, fundamental study of microwave technical and design of microwave filter. E-mail: dvphuongntcity@gmail.com; phuongdovan@tcu.edu.vn

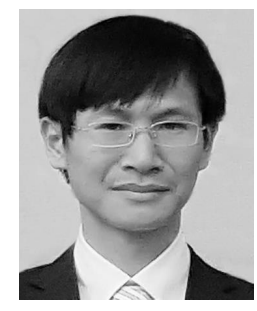

Duy-Manh Luong received the B.S. and M.S. degrees in physics from Hanoi University of Science (HUS), a member of Vietnam National. University (VNU), Hanoi, Vietnam, in 2005 and 2007, respectively, and the D.E. degree in electronics engineering from the University of ElectroCommunications (UEC), Tokyo, Japan, in March 2016. He is currently a specially appointed researcher at Graduate School of Engineering Science, Osaka University, Japan. His research interests include development of microwave semiconductor devices and circuits and millimeterwave ( $\mathrm{mmW}$ ) systems for wireless communication applications based on resonant tunneling diodes (RTDs) and photonic crystals.

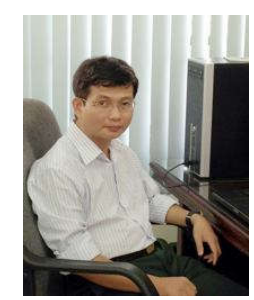

Chi-Hieu Ta was born in Vinh Phuc in 1970. He graduated the Military Technical Academy in 1994 with hornor. He got his MSc degree in electronic engineering in the National Defense Academy of Japan in 2002 and his PhD degree in signal processing in the University of Strathclyde, United Kingdom in 2008. He is currently working at the Faculty of Radio Electronics, Military Technical Academy. His research interests include precoding and equalization for MIMO systems, microwave engineering and computational electromagnetics.

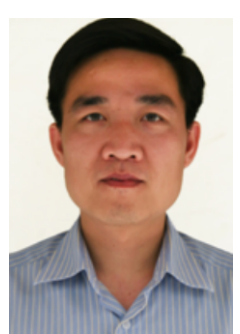

Minh-Tan Doan received the B.Eng and M.Eng degrees in Radio Elictronic Engineering from Le Qui Don Technical University, Hanoi, Vietnam in 2000 and 2003, respectively, Ph.D. degree in Department of Communication Engineering Nanjing University of Science and Technology, Nanjing, China in 2012, He is currently a lecturer of Faculty of Radio-Electronics Engineering, Le Qui Don Technical University, Hanoi. His research interests include the design of microwave filter and associated RF modules for microwave and signal processing for communication. 